Article

\title{
Immortalization of Porcine 11 $\beta$-Hydroxysteroid Dehydrogenase Type 1-Transgenic Liver Cells Using SV40 Large T Antigen
}

\author{
Hee Young Kang 1,3 (D), Young-Kwon Choi ${ }^{1}$, Yeon Ik Jeong ${ }^{2}$, Kyung-Chul Choi ${ }^{1}$, \\ Sang-Hwan Hyun ${ }^{1}$, Woo-Suk Hwang ${ }^{2}$ and Eui-Bae Jeung ${ }^{1, *}$ (1D \\ 1 College of Veterinary Medicine, Chungbuk National University, 1 Chungdae-ro, Seowon-gu, Cheongju, \\ Chungbuk 28644, Korea; heeyoung.kang@daum.net (H.Y.K.); cyk901124@naver.com (Y.-K.C.); \\ kchoi@chungbuk.ac.kr (K.-C.C.); shhyun@cbu.ac.kr (S.-H.H.) \\ 2 Sooam Biotech Research Foundation, 64 Kyunginro, Guro-gu, Seoul 08359, Korea; \\ youniks@sooam.org (Y.I.J.); hwangws@sooam.org (W.-S.H.) \\ 3 Immunotherapy Convergence Research Center, Korea Research Institute of Bioscience and \\ Biotechnology (KRIBB), 125 Gwahak-ro, Yuseong-gu, Daejeon 34141, Korea \\ * Correspondence: ebjeung@chungbuk.ac.kr; Tel.: +82-43-261-2397
}

Received: 24 September 2017; Accepted: 2 December 2017; Published: 5 December 2017

\begin{abstract}
Cortisol is a steroid hormone essential to the maintenance of homeostasis that is released in response to stress and low blood glucose concentration. Cortisol is converted from cortisone by $11 \beta$-hydroxysteroid dehydrogenase type 1 (HSD11B1). It has been reported that too much cortisol or overexpression of HSD11B1 induces obesity and the insulin resistance that accompanies metabolic syndrome in rodent adipose tissue. In our previous study, HSD11B1-transgenic (TG) fibroblasts were established, and a porcine model was generated by SCNT using those fibroblasts. Hepatocytes overexpressing HSD11B1 were obtained from livers of this porcine model and cultured in vitro. However, the primary hepatocytes were found to have a short life span or low proliferation rate. To overcome these problems, the SV40 large T antigen was transduced into primary HSD11B1-TG hepatocytes, and those cells were immortalized. Immortalized HSD11B1-TG hepatocytes showed restored morphology, more rapid proliferation rate, and more expression of HSD11B1 than primary hepatocytes. As well, these cells kept the hepatic characteristics such as gluconeogenic response to cortisone and increased expression of hepatic makers. The immortalized HSD11B1-TG hepatocytes may be useful for studying traits and potential therapeutic drugs for treatment of metabolic disorders induced by overexpression of HSD11B1.
\end{abstract}

Keywords: pig; hepatocytes; immortalization; HSD11B1

\section{Introduction}

The liver plays a major role in metabolism including regulation of glycogen storage, protein synthesis, hormone production and detoxification of various metabolites. The liver primarily consists of hepatocytes, which contribute to the major functions of liver and are a principal target of cortisol. A study of Clinical Endocrinology reported a direct connection between cortisol levels in men and fatty liver disease [1]. According to that study, nonalcoholic fatty liver disease patients had chronic overactivity in the hypothalamo-pituitary-adrenal axis, which led to a subclinical version of Cushing syndrome and the overproduction of cortisol [1].

$11 \beta$-hydroxysteroid dehydrogenase type 1 (HSD11B1) is a nicotinamide adenine dinucleotide phosphate (NADPH)-dependent enzyme located within the lumen of the endoplasmic reticulum (ER) [2] that is highly expressed in metabolic tissue including that of the liver, adipose tissue, and the 
central nervous system [3]. HSD11B1 acts as a reductase, converting cortisone into cortisol $[4,5]$. In the early fasting state, cortisol causes gluconeogenesis or glycogenolysis in liver and muscle, and activates anti-stress and anti-inflammatory pathways. Cortisol also stimulates the activation of glycogen phosphorylase, which is necessary for epinephrine to have an effect on glycogenolysis [6]. Hsd11b1-transgenic (TG) mice show abdominal obesity, hyperglycemia, insulin resistance, hyperphagia, hyperleptinemia and increased intra-adipose and portal levels, but not systemic corticosterone levels [7].

In our study, HSD11B1-TG male pigs were generated as a disease model for metabolic syndrome by somatic cell nuclear transfer (SCNT) using recloned fibroblasts. Recloned fibroblasts were primary cultured cells obtained from the first male pig generated via SCNT using transgenic porcine fibroblasts that had been established in our laboratory in 2013 [7]. Since HSD11B1 has been associated with several metabolic disorders, it has been investigated as a novel target for potential therapeutic drugs [8]. Thus, HSD11B1-TG hepatocytes will be useful for studying metabolic diseases such as hyperglycemia and fatty liver disease. However, establishment of a HSD11B1-TG hepatic cell line has not been reported to date. In this study, hepatocytes obtained from HSD11B1-TG male pigs were cultured. These primary hepatocytes could be maintained for several days and until 10 passages; however, their proliferation rate decreases, and eventually stops with morphological change [9].

This blockage of cell proliferation can be overcome by SV40 oncogene large T antigen (SV40LT), which inactivates both p53 and retinoblastoma [10]. Cell immortalization is accomplished through a two-stage process. Specifically, cells expressing SV40LT escape senescence but continue to lose telomeric repeats during their extended life span. Eventually, terminal telomere shortening causes the cells to reach a second non-proliferative stage termed "crisis" [11]. Escape from crisis is a very rare event accompanied by the reactivation of telomerase [12].

In this study, we introduced SV40LT into primary HSD11B1-TG (Pri11ßTG) hepatocytes to establish immortalized cells in vitro. Since SV40LT-HSD11B1-TG (SV11ßTG) hepatocytes contain oncogenes, these cells proliferate well under in vitro conditions. We then investigated whether the SV11ßTG hepatic cell line kept the original characteristics of hepatocytes and if the morphology of immortalized hepatocytes was recovered.

\section{Results}

\subsection{Confirmation of Altered Morphology and Genomic Hybridization of SV40LT in SV11ßTG Hepatocytes}

Pri11ßTG hepatocytes were isolated from the liver of HSD11B1-TG male pigs and immortalized through SV40LT transduction. One day after the cell isolation, the hepatocyte was weakly adherent and showed a small round shape (Figure 1A). After 4 days, the cells were strongly adhered and started to grow (Figure 1B). Morphology of Pri11ßTG hepatocytes became larger and longer as the passage progressed (Figure 1C). On the other hand, SV11 $\beta$ TG hepatocytes became smaller as passage progressed, and were similar to primary hepatocytes with passage 0-2 (Figure 1B,E). Immortalized SV11 $\beta$ TG hepatocytes have recovered hepatic morphology and size at approximately passage 35 (Figure 1D-F). PCR was then performed using primer sets specific for the vector to confirm the chromosomal integration of the targeting vector (Table 1). The SV40LT gene was only observed in genomic DNA of immortalized SV11 $\beta$ TG hepatocytes (Figure 1G). The HSD11B1 cassette and selection cassette were confirmed in genomic DNA of both Pri11 $\beta$ TG and SV11 $\beta$ TG hepatocytes (Figure 1H,I). Both Pri11 $\beta$ TG and SV11 $\beta$ TG hepatocytes express EGFP due to the selection cassette with the EGFP gene (Figure 1F).

\subsection{Expression of SV40LT $m R N A$ and Increase of Cell Proliferation Rate in SV11ßTG Hepatocytes}

To confirm that mRNA of SV40LT is stably expressed, reverse transcription using total RNA and PCR was performed. Expression of SV40LT mRNA was only observed in SV11ßTG hepatocytes (Figure 2A). After immortalization by SV40LT, SV11 $\beta$ TG hepatocytes grew more rapidly and showed greater proliferation than Pri11 $\beta$ TG hepatocytes. A CCK-8 assay was carried out to assess the difference 
in proliferation rate (Figure 2B). The line slope for absorbance to time (h) is 0.0062 in Pri11ßTG hepatocytes, while it is 0.0095 in SV11 $\beta$ TG hepatocytes. The proliferation rates of SV11 $\beta$ TG hepatocytes were significantly (1.53 times) faster than those of Pri11ßTG hepatocytes

Table 1. Primer sequences used in polymerase chain reaction (PCR).

\begin{tabular}{|c|c|c|c|}
\hline Primer & Genebank ID & & Sequences $\left(5^{\prime}\right.$ to $\left.3^{\prime}\right)$ \\
\hline \multirow{2}{*}{ HSD11B1 } & \multirow{2}{*}{ NM_214248 } & $\mathrm{F}$ & CAACGTGTCAATCACGCTCT \\
\hline & & $\mathrm{R}$ & TTCCTGGATTTTCCAACAGG \\
\hline \multirow{2}{*}{ H6PD } & \multirow{2}{*}{ XM_005674044 } & $\mathrm{F}$ & CAGGCTGGAGGAGTTCTTTG \\
\hline & & $\mathrm{R}$ & AGGTCTCGCGGATGTTCTT \\
\hline \multirow{2}{*}{ G6PT } & \multirow{2}{*}{ GU207843 } & F & CTGTCTCCTGCTCATCCACA \\
\hline & & $\mathrm{R}$ & AGAACTGGCCCCAGTCAGTA \\
\hline \multirow{2}{*}{ G6PC } & \multirow{2}{*}{ EU717834 } & $F$ & AAGTTGTTGCTGGGGTCTTG \\
\hline & & $\mathrm{R}$ & CCTTCGCTTGGCTTTCTCTA \\
\hline \multirow{2}{*}{ PCK1 } & \multirow{2}{*}{ NM_001123158 } & $\mathrm{F}$ & GAGCACAAGGGCAAAGTGATTAT \\
\hline & & $\mathrm{R}$ & GAGCCAGTGGGCCAGGTATT \\
\hline \multirow{2}{*}{ GR } & \multirow{2}{*}{ NM_001008481 } & $\mathrm{F}$ & AGCATGCCGCTACAGAAAGT \\
\hline & & $\mathrm{R}$ & GACTTCCAGCAGTGACACCA \\
\hline \multirow{2}{*}{ ALB } & \multirow{2}{*}{ NM_001005208 } & $F$ & TGTCTTCCTGGGCACGTTTT \\
\hline & & $\mathrm{R}$ & TAGGCTCATCCACAAGAGGC \\
\hline \multirow{2}{*}{ AFP } & \multirow{2}{*}{ NM_214317 } & $\mathrm{F}$ & TGCTTTCAAACAAAGGCAGCA \\
\hline & & $\mathrm{R}$ & ACTCCAGCACGTTTCCTCTG \\
\hline \multirow{2}{*}{ SERPINA1 } & \multirow{2}{*}{ NM_214395 } & $\mathrm{F}$ & ACCCAAGTTCTGCCAATCTACA \\
\hline & & $\mathrm{R}$ & GTGGCCTCTGTCCCTTTCTC \\
\hline \multirow{2}{*}{ RN18S } & \multirow{2}{*}{ NR_046261 } & $\mathrm{F}$ & CGCGGTTCTATTTTGTTGGT \\
\hline & & $\mathrm{R}$ & AGTCGGCATCGTTTATGGTC \\
\hline \multicolumn{4}{|c|}{ Primers to Confirm Genomic Integration } \\
\hline \multirow{2}{*}{ HSD11B1 cassette } & & $\mathrm{F}$ & CCATGATAATAAGCCTGCTCTACTCCA \\
\hline & & $\mathrm{R}$ & GGAAGTCATGAAGGCCTGGGTGATG \\
\hline \multirow{2}{*}{ Selection cassette } & & $\mathrm{F}$ & CATGAAGCAGCACGACTTCT \\
\hline & & $\mathrm{R}$ & CCTAGGAATGCTCGTCAAGA \\
\hline \multirow{2}{*}{ SV40LT } & \multirow{2}{*}{ NC_001669 } & $\mathrm{F}$ & CTGACTTTGGAGGCTTCTGG \\
\hline & & $\mathrm{R}$ & GGAAAGTCCTTGGGGTCTTC \\
\hline \multicolumn{4}{|c|}{ Primers to Detect miR-122 and RNU43 } \\
\hline \multirow{2}{*}{ miR-122 } & & $\mathrm{F}$ & TGGAGTGTGACAATGGTGTTTG \\
\hline & & $\mathrm{R}$ & AACGCCATTATCACACTAAATA \\
\hline \multirow{2}{*}{ RNU43 [13] } & & $\mathrm{F}$ & GTGAACTTATTGACGGGCG \\
\hline & & $\mathrm{R}$ & GTGCAGGGTCCGAGGT \\
\hline
\end{tabular}

2.3. Overexpression of HSD11B1 mRNA but No Significant Changes of H6PD and G6PT mRNA in SV11ßTG Hepatocytes

It has been reported that cortisol production is catalyzed via the triad system, which consists of glucose-6-phosphate transporter (G6PT or SLC37A4), hexose-6-phosphate dehydrogenase (H6PD) and HSD11B1 in ER of hepatocytes, and that the reductase activity of HSD11B1 was activated by NADPH generated via H6PD $[2,14]$. HSD11B1 showed more expression in SV11 $\beta$ TG than Pri11ßTG hepatocytes (Figure 2C). Although HSD11B1 increased significantly, the expression level of H6PD and G6PT did not change significantly (Figure 2D,E). These results indicate that the overexpression of $H S D 11 B 1$ does not directly affect the expression of H6PD and G6PT.

\subsection{Verification of Functional Hepatocytes through Expression of Gluconeogenic Markers}

Cortisol converted by HSD11B1 facilitates hepatic gluconeogenesis. To identify the effects of HSD11B1 overexpression on gluconeogenic genes including glucose-6 phosphatase catalytic subunit 
(G6PC, Figure 3A) and phosphoenolpyruvate carboxykinase 1 (PCK1, Figure 3B), we measured mRNA levels by real-time PCR and PCK1 protein levels by Western blotting (Figure 3D). The mRNA expression of G6PC and PCK1 increased significantly in response to treatment with cortisone, the substrate of HSD11B1, in both Pri11ßTG and SV11ßTG hepatocytes (Figure 3A,B), while no difference in expression of either gene was observed in dimethyl sulfoxide (DMSO) treated cells, as vehicle control. Similar to the mRNA level, expression of HSD11B1 protein in SV11ßTG hepatocytes was greater than in Pri11 $\beta$ TG hepatocytes (Figure 3D,F). Moreover, expression of PCK1 protein increased slightly under cortisone treatment (Figure 3D,E). Also, we examined whether overexpression of HSD11B1 induces the expression of glucocorticoid receptor (GR, Figure 3C), which binds to cortisol or other glucocorticoids and acts as a effector in the cortisol-mediated response $[15,16]$. The level of $G R$ mRNA was meaningfully increased in cortisone-treated condition of SV11 $\beta$ TG hepatocytes, similar to increasing pattern of HSD11B1 mRNA and protein. These findings suggest that transgenic HSD11B1 acts as a functional reductase, is not affected by the immortalization process, and that SV11ßTG hepatocytes retain the traits of Pri11ßTG hepatocytes.

A

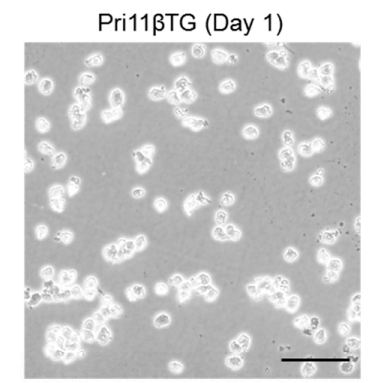

D

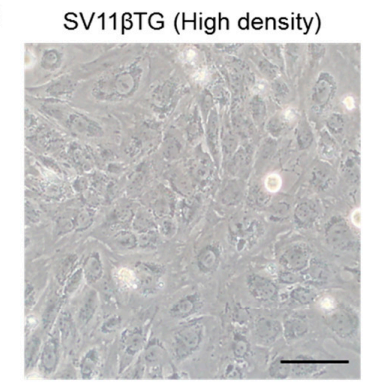

G

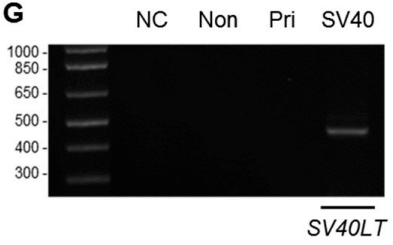

B

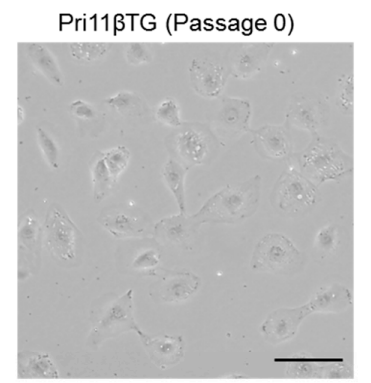

E

SV11ßTG (Low density)
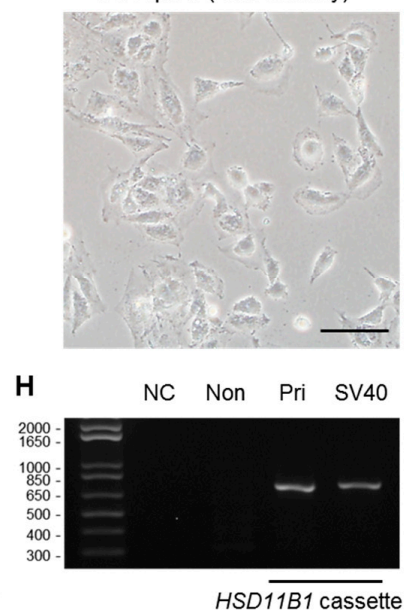

c

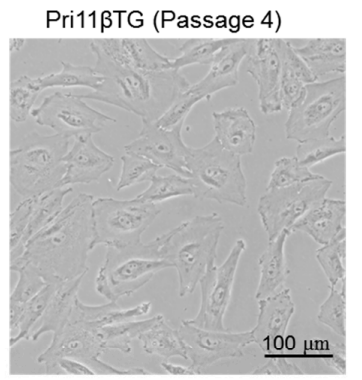

F
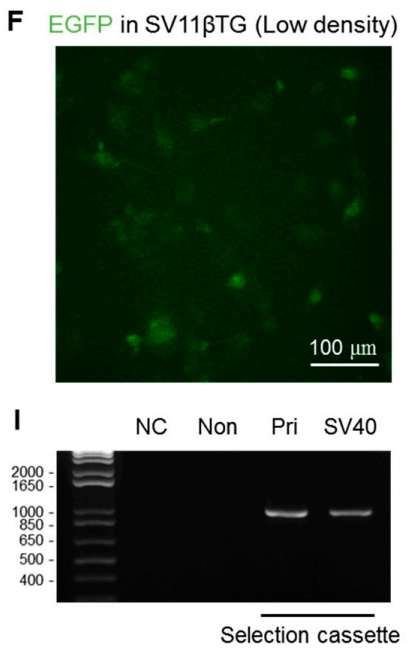

Figure 1. Morphological change and PCR-based confirmation of genomic integration in immortalized SV11 $\beta$ TG hepatocytes. (A-C) Accordance with the progression of passages (one day after cell isolation (A), the zero passage (B), and the fourth passage (C)), morphology of Pri11ßTG hepatocytes became longer and fibroblast-like, and the size of hepatocytes increased; (D-F) Morphology and size of the SV11 $\beta$ TG hepatocytes, which were immortalized by SV40 large T antigen, recovered similar to Pri11 $\beta$ TG ones at the first passage in high density (D) and low density (E); EGFP expression (F) was confirmed in 11ßTG hepatocytes due to the selection cassette including EGFP and neomycin resistance gene $\left(\mathrm{Neo}^{r}\right)$ [7] (Scale bar, $\left.100 \mu \mathrm{m}\right)$; (G-I) Chromosomal insertion was confirmed by PCR using primers flanking the target cDNA in SV40LT sequences (G) for immortalization, in HSD11B1 expression cassette $(\mathbf{H})$ and in selection cassette (I). (NC, negative control without template; Non, non-transgenic hepatocytes; Pri, Pri11 $\beta$ TG hepatocytes; SV40, SV11 $\beta T G$ hepatocytes; SV40LT, SV40 large $\mathrm{T}$ antigen). 
A

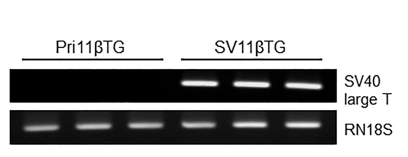

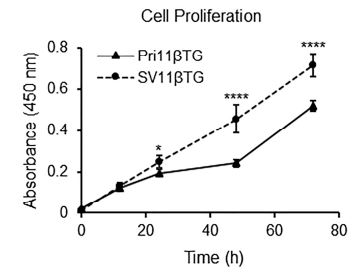
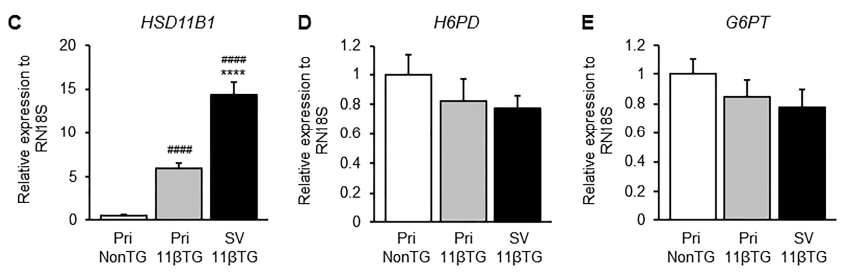

Figure 2. Cell-proliferation rate in SV11 $\beta$ TG hepatocytes expressing SV40LT and effect of HSD11B1 overexpression on H6PD and G6PT mRNA in hepatic G6PT-H6PD-HSD11B1 triad system. (A) The mRNA expression of SV4LT was observed in immortalized SV11 $\beta T G$ hepatocytes by PCR; (B) The proliferation rate of Pri11ßTG $(\boldsymbol{\Delta})$ and SV11ßTG $(\bullet)$ hepatocytes for three days was measured by CCK-8 assay. The rate was significantly elevated in SV11ßTG hepatocytes. Each group was prepared to half-dozen wells, and this experiment was performed twice; (C) Expression of HSD11B1 was highly increased; (D,E) Expression of H6PD (D) and G6PT (E) was insignificantly changed by transduction of HSD11B1 gene and immortalization using SV40LT antigen. Relative expression levels of mRNA were investigated by qPCR and normalized to RN18S. These experiments were performed in triplicate and independently three times. Number signs and asterisks indicate significant difference comparing to PriNonTG ( $\left.{ }^{\# \# \# ~} p<0.0001\right)$ and Pri11ßTG $\left(^{*} p<0.05,{ }^{* * * *} p<0.0001\right)$ hepatocytes, respectively. White, gray and black bars represent PriNonTG, Pri11 $\beta$ TG and SV11 $\beta$ TG hepatocytes, respectively.
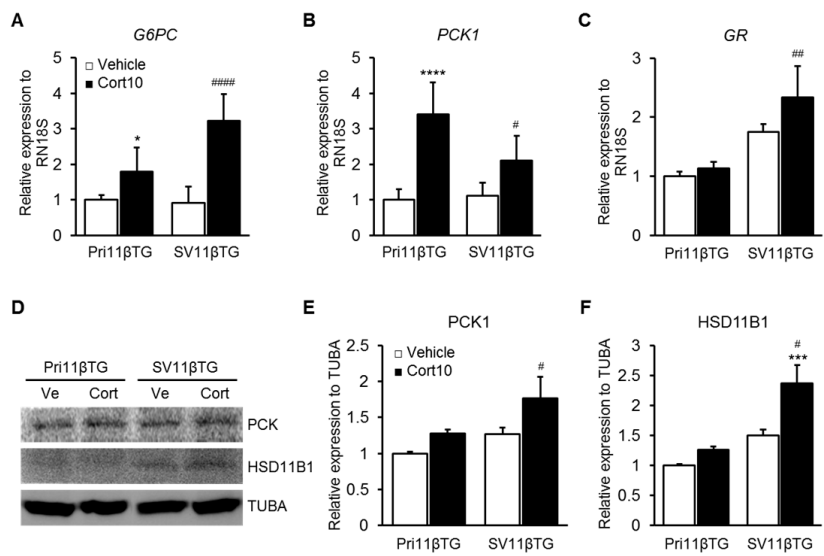

Figure 3. Induction of gluconeogenic markers by cortisone treatment. (A,B). The mRNA levels of gluconeogenesis-related genes such as G6PC (A) and PCK1 (B) were significantly increased in cortisone-treated group of both Pri11ßTG and SV11ßTG hepatocytes; (C) The mRNA level of GR

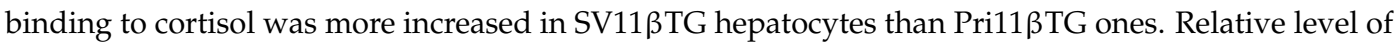
these mRNAs was normalized to RN18S; (D) protein level of PCK1 and HSD11B1 was examined by Western blotting method; (E,F) Relative expression of these protein was quantified by ImageJ program and normalized to $\alpha$-tubulin. Both PCK1 and HSD11B1 proteins levels meaningfully increased in cortisone-treated condition of SV11 $\beta$ TG hepatocytes. Asterisks and number signs respectively mean significant increase comparing to vehicle-treated group of Pri11ßTG $\left({ }^{*} p<0.05\right.$, ${ }^{* * *} p<0.001$ and **** $p<0.0001)$ and SV11ßTG $\left({ }^{\#} p<0.05,{ }^{\# \#} p<0.01\right.$ and $\left.{ }^{\# \# \#} p<0.0001\right)$ hepatocytes. All experiments were performed independently at least three times. White bars represent vehicle and black bars represent cortisone-treated group. (Ve, vehicle; Cort, cortisone) 


\subsection{Confirmation of Hepatic Character Using Liver Markers}

To identify the hepatic traits of cultured hepatocytes, we measured mRNA levels of liver specific genes such as albumin $(A L B$, Figure $4 \mathrm{~A}), \alpha$-fetoprotein $(A F P$, Figure $4 \mathrm{~B})$ and serpin peptidase inhibitor $\alpha 1$-antitrypsin member 1 (SERPINA1, Figure 4C), and confirmed expression of ALB protein (Figure 4D). Expression of ALB protein as well as ALB and SERPINA1 mRNA was identified in SV11 $\beta$ TG cells and, increased significantly than in Pri11 $\beta$ TG cells (Figure 4A,C,D). It means that immortalized SV11 $\beta$ TG cells should be hepatocytes. Moreover, mRNA levels of AFP and SERPINA1 were up-regulated in both Pri11ßTG and SV11ßTG hepatocytes by treatment with $10 \mu \mathrm{M}$ cortisone (Figure 4B,C). The liver-enriched microRNA-122 (miR-122) level in SV11ßTG hepatocytes was similar to one in HepG2, and was more expressed than in Pri11ßTG hepatocytes (Figure 4E). These results suggest that SV11ßTG hepatocytes recover, or retain the characteristics of hepatocytes and are useful for the functional hepatocytes.

A

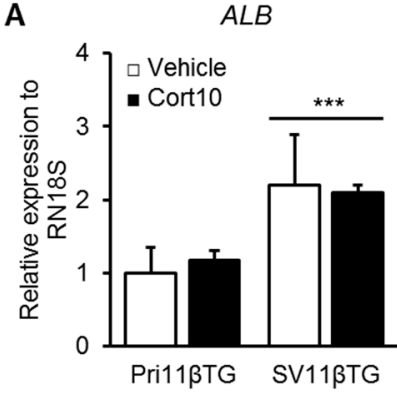

D
B

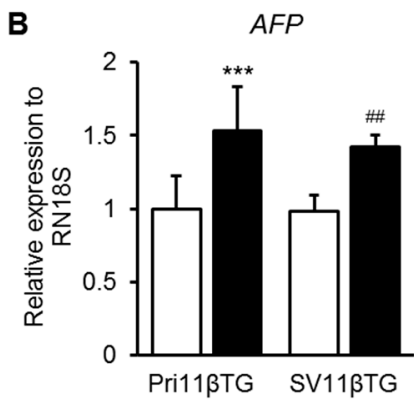

E
C

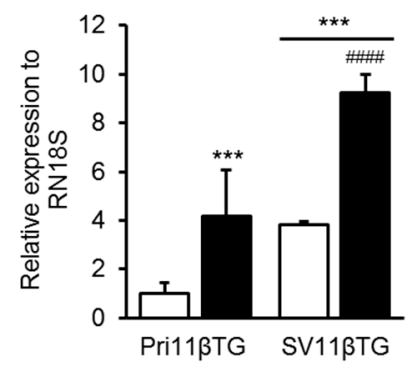

$\operatorname{miR}-122$
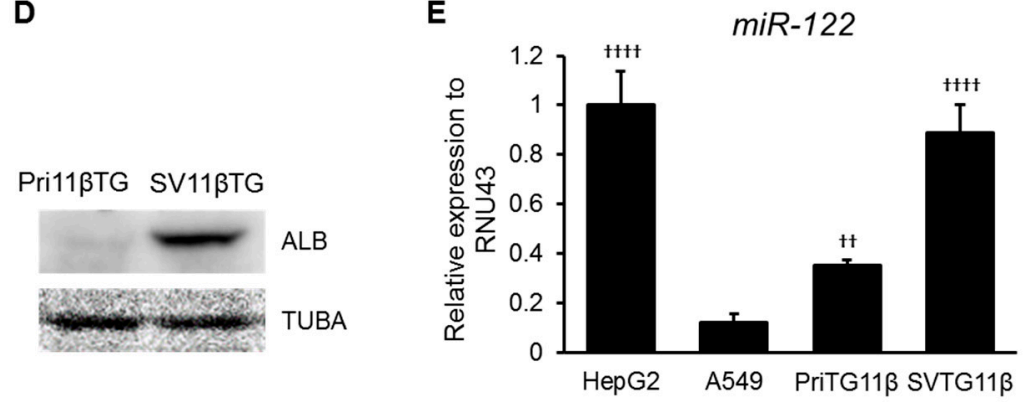

Figure 4. Identification of hepatic markers. (A-C) Whether Pri11ßTG and SV11 $\beta$ TG hepatocytes keep hepatic traits was examined by qPCR using primer to hepatic markers including $A L B$ (A), $A F P(\mathbf{B})$, and SERPINA1 (C), (A,C); The mRNA levels of $A L B$ and SERPINA1 was significantly elevated in SV11ßTG hepatocytes; (B,C) AFP and SERPINA1 mRNA levels were meaningfully increased in cortisone-treated group. Asterisks and number signs mean significant increase comparing to

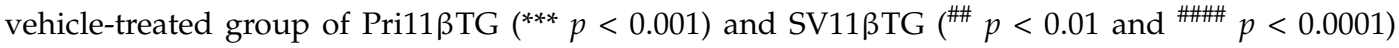
hepatocytes respectively. Relative mRNA level was normalized to RN18S. These experiments were repeated independently three times. White bars represent vehicle and black bars represent cortisone-treated group; (D,E) Hepatic markers including ALB protein (D) and miR-122 (E) was identified and increased in SV11ßTG hepatocytes. A549, a lung cancer cell line, was used as a negative control. Daggers indicate significant difference comparing to A549 cell line $\left(\left(^{++} p<0.01\right.\right.$ and $\left.{ }^{++t+} p<0.0001\right)$. The relative miRNA level was normalized to RNU43. These experiments were repeated twice in triplicate.

\section{Discussion}

Since pigs are mono-gastric omnivores and have anatomical and physiological characteristics highly comparable to those of humans, porcine models are suitable for investigating and understanding the biological pathways related to human metabolic diseases [17]. In this study, we established 
immortalized porcine HSD11B1-TG (SV11ßTG) hepatocytes, which are useful for investigation of metabolic disorders, through transduction of SV40LT antigen. In these immortalized cells, we confirmed integration of the HSD11B1 gene cassette, selection cassette with the Neo ${ }^{r}$ and GFP genes, and the SV40LT gene.

HSD11B1 mRNA and protein were overexpressed in SV11ßTG hepatocytes; hence, the expression of G6PT and H6PD, which act as triads with HSD11B1 in ER, has no significant effect on mRNA level. These results may have been attributed to different basal expression levels of the three genes. The expression level of H6PD was 50-60 times higher than HSD11B1 and that of G6PT was 7-9 times higher than HSD11B1. G6PT enzyme is a transmembrane protein consisting of three transporting subunits (G6PT1, G6PT2 and G6PT3) that provides a selective channel and transports glucose-6-phosphate produced in the terminal reactions of both glycogenolysis and gluconeogenesis from the cytosol into the lumen of the ER. During this process, H6PD produces the cofactor NADPH for HSD11B1 activation [2]. Activated HSD11B1 converts inactive cortisone into active cortisol, which increases the glucose level in blood through gluconeogenesis and is involved in the metabolism of fat, protein, and carbohydrates [6]. Increased level of GR mRNA may indicate the increase in cortisol converted by HSD11B1 in SV11 $\beta$ TG hepatocytes.

In a study using $d b / d b$ mice, it has been reported that increased expression of GR and HSD11B1 in hepatocytes is an important component in the development of type 2 diabetes [18]. Also, hepatic GR deficiency by delivering an adenovirus expressing GR-specific shRNA improves hepatic steatosis in livers of $d b / d b$ mice [19]. The GR has been characterized as a crucial regulator of glucose homeostasis [19], belongs to nuclear receptor subfamily 3, group C, member1 (NR3C1) and binds to cortisol [14]. Complex consisting of GR and cortisol regulates transcription of genes related to the development, metabolism and immune response [16]. In this study, this complex has induced the expression of gluconeogenic and downstream genes such as G6PC and PCK1. However, overexpression of HSD11B1 did not induce the increase of G6PT and H6PD expression in the hepatic G6PT-H6PD-HSD11B1 triad system.

To verify the hepatic traits of SV11 $\beta$ TG hepatocytes, we examined the expression of liver markers including ALB, AFP, SERPINA1, and miR-122. Most of these markers increased in immortalized hepatocytes. ALB protein is made specifically in the liver [20]. Primary cultured cells lost the inherent properties and morphology of original tissue in accordance with the progression of passages. Increased expression of ALB protein in SV11 $\beta$ TG hepatocytes indicates that SV11ßTG hepatocytes had recovered from the loss of tissue specificity. It is also supported by the fact that miR-122, which is necessary for the regulation of liver development, differentiation and metabolic function [21], has been further increased in SV11 $\beta$ TG hepatocytes than in Pri11 $\beta$ TG hepatocytes. The temporal regulation of miR-122 has been reported to promote hepatobiliary segregation with the acquisition and maintenance of hepatocyte phenotype, and to be involved in the regulation of cholesterol and fatty acid metabolism [21]. In some murine studies, genetic deletion of $m i R-122$ showed severe dysfunction on lipid metabolism, and micro-steatosis, fibrosis and inflammation in the liver [22,23].

AFP was relatively highly expressed in the fetal liver [24], and AFP level in the serum decreased in the weeks and months after birth [25]. As shown in Figure 4, AFP level did not differ between Pri11 $\beta$ TG and SV11 $\beta$ TG, but increased in response to treatment with cortisone. Some studies have reported that temporary treatment of cortisol or dexamethasone in hepatic tumor reduces AFP [26,27]. However, in a 10-year-old boy with pancreatic acinar cell tumor, Cushing's syndrome, which was diagnosed with high serum adrenocorticotropic hormone and cortisol levels, was identified with the tumor, and excessive AFP level in serum was observed [28]. This clinical result differs from other studies in that it is continuously exposed to cortisol, and HSD11B1 is expected to be highly expressed in the patient, considering the increase of AFP expression in cortisone treatment in our 11ßTG hepatocytes. Also, an elevated AFP concentration in the serum of adults was primarily observed in patients with hepatocellular carcinoma, chronic hepatitis, and acute liver failure [29]. Considering the influence 
of cortisol on hepatic metabolism, these findings suggest that cortisol can affect AFP levels during metabolic stress responses.

Since SERPINA1 is expressed in the liver [30], it was used to confirm hepatic traits. SERPINA1 is one of the most abundant serine protease inhibitors belonging to the serpin superfamily and is known by other names such as $\alpha 1$-antitrypsin (A1AT) and $\alpha 1$ protease inhibitor (A1PI) because of its ability to inhibit various proteases [31]. SERPINA1 is capable of increasing greatly upon acute inflammation. SERPINA1 plays a key role in protection of tissues from protease of inflammatory cells, especially neutrophil elastase [32]. Deficiency and mutation of SERPINA1 can cause pulmonary emphysema via uncontrolled elastase activity and liver cirrhosis through accumulation of misfolded proteins and impaired secretion [33]. Therefore, the use of three SERPINA1 products obtained from human plasma, Prolastin, Zemaira, and Aralast, was approved by the United States Food and Drug Administration [34]. It has been reported that treatment with SERPINA1 products significantly reduced neutrophils, TNF- $\alpha$, and neutrophil chemokine KC (CXCL1) [35]. Hence, it was reported that cortisol, a steroid hormone, suppresses the immune system by preventing production of IL12, IFN $\gamma$, IFN $\alpha$ and TNF $\alpha$ via antigen presenting cells and T helper cells [36]. As shown in Figure 4, the levels of SERPINA1 increased in immortalized SV11ßTG hepatocytes and were significantly elevated in response to treatment with cortisone. These findings suggest that cortisol increases the expression of SERPINA1, and hepatic traits in SV11ßTG hepatocytes had been restored.

In conclusion, we used the SV40LT system for immortalization. Although human liver sinusoidal endothelial cells have previously been immortalized using the hTERT gene [37], previous studies have never reported immortalization of porcine HSD11B1-TG hepatocytes by introduction of SV40LT in vitro. Furthermore, these cells have a tendency to maintain their original characteristics, such as response to cortisone and gluconeogenesis. Overexpression or dysregulation of HSD11B1 has been associated with metabolic disorders eliciting abdominal obesity, hyperglycemia, hyperphagia, hyperleptinemia, and insulin resistance. Recently, HSD11B1 has been investigated as a novel target of potential therapeutic drugs for metabolic syndrome, including Cushing syndrome. Thus, our immortalized SV11 $\beta$ TG hepatocytes may be useful for investigating traits and potential therapeutic drugs for metabolic disorders.

\section{Materials and Methods}

\subsection{Animals and Primary Porcine Hepatocytes Culture}

The pig experiments were performed in strict accordance with the recommendations in the Guide for the Care and Use of Laboratory Animals of the National Veterinary and Quarantine Service, and this study was approved by the animal ethics committee of Sooam Biotech Research Foundation (P-15-01) and the Committee on the Ethics of Animal Experiments of the Chungbuk National University (CBNUA-871-15-01). All efforts were made to minimize animal suffering.

\subsection{Isolation of Primary Porcine Hepatocytes}

Liver was removed from Yucatan male pig, washed in cold PBS, chopped on cold petri dish, and dispersed with $0.05 \%$ trypsin at $37^{\circ} \mathrm{C}$ for $15 \mathrm{~min}$. Supernatant was mixed to the same volume of basal medium (Dulbecco Modified Eagle Medium (DMEM) with $25 \mathrm{mM}$ glucose, $3.7 \mathrm{~g} / \mathrm{L}$ sodium bicarbonate ( $\mathrm{pH} 7.4$ ), 10\% fetal bovine serum (FBS), $100 \mathrm{U} / \mathrm{mL}$ penicillin, $100 \mu \mathrm{g} / \mathrm{mL}$ streptomycin, and $5 \mu \mathrm{g} / \mathrm{mL}$ plasmocin) and centrifuged (1200 rpm for $5 \mathrm{~min}$ ). The pellet was resuspended in $4 \mathrm{~mL}$ of basal medium and layered over a percoll gradient in phosphate-buffered saline (PBS) containing 70 to $5 \%$ percoll (vol/vol). After centrifugation for $20 \mathrm{~min}$ at $3600 \mathrm{rpm}$, the middle layer among three regions was collected. The collected cell was cultured in basal media and in a humidified $5 \% \mathrm{CO}_{2}$ atmosphere at $37^{\circ} \mathrm{C}$. 


\subsection{Cell Treatments}

Pri11ßTG or SV11 $\beta$ TG hepatocytes $\left(1 \times 10^{5}\right.$ cells per well $)$ were attached for one day, then starved in starvation medium (DMEM with $5 \mathrm{mM}$ glucose without FBS and phenol-red) for one day before being treated with $0.1 \%$ DMSO or $10 \mu \mathrm{M}$ cortisone in starvation medium containing $10 \%$ charcoal-dextran treated FBS for two days.

\subsection{Immortalization of Primary Hepatocytes}

To introduce SV40LT into primary cell, retroviral vector pLXIN-SV40T was stably transfected into the recombinant retrovirus packaging cell line, PT67 cells (Takara, Shiga, Japan). Retrovirus-containing medium was collected, and transferred onto Pri11ßTG hepatocytes [38].

\subsection{Genomic DNA Extraction and Confirmation of SV40T Integration}

Genomic DNA was isolated with a G-DEX ${ }^{\mathrm{TM}}$ IIc Genomic DNA Extraction kit (iNtRON, Gyeonggi-do, South Korea). $100 \mathrm{ng}$ of genomic DNA was amplified in a $20 \mu \mathrm{L}$ PCR reaction containing $1 \mathrm{U}$ i-Start Taq polymerase (iNtRON), $2 \mathrm{mM}$ dNTPs (Takara) and 10 pmol of each specific primer listed in Table 1. Amplicons were separated on $1 \%$ or $1.5 \%$ agarose gel, stained with ethidium bromide, photographed under UV illumination, and scanned using GelDoc EQ (Bio-Rad, Hercules, CA, USA).

\subsection{Cell Counting Kit-8 (CCK-8) Assay}

The number of cells on 96 well-plate was seeded to $4 \times 10^{3}$ cells per well. The cell was maintained in basal medium and cultured for 10, 28, 48, and $72 \mathrm{~h}$. These cells were incubated with CCK-8 solution (Dojindo, Rockville, MD, USA) for $2 \mathrm{~h}$. Absorbance of each well was measured at $450 \mathrm{~nm}$.

\subsection{RNA Isolation and Gene Expression Analysis by RT-PCR and $q P C R$}

Total RNA was extracted using TRI Reagent (Thermo Fisher Scientific, Carlsbad, CA, USA) according to the manufacturer's instructions. cDNA was synthesized using $M M L V$ reverse transcriptase (Thermo Fisher Scientific) and random primers (TaKaRa; 9-mers) or stem-loop RT primer (miR-122, GTTGGCTCTGGTGCAGGGTCCGAG GTATTCGCACCAGAGCCAACAACGCC [39]; RNU43, GTTGGCTCTGGTGCAGGGTCCGAGGT ATTCGCACCAGAGCCAACAATCAG [13]). Prime Q-master mix (GeNet Bio, Daejeon, South Korea) was used for quantitative PCR (qPCR). Primer sequences of target genes are described in the Table 1. Relative expression mRNA and miRNA

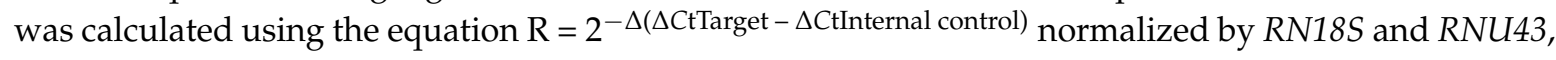
respectively. RNU43, which called the small-nucleolar RNA, C/D Box 43 (SNORD43), belongs to the large $\mathrm{C} / \mathrm{D}$ box family and function in 2'-O-ribose methylation in ribosome biogenesis.

\subsection{Protein Expression Analysis by Western Blotting}

Lysates were extracted in RIPA buffer (50 mM Tris, pH 7.4, $150 \mathrm{mM}$ sodium chloride ( $\mathrm{NaCl}$ ), $1 \%$ Triton X-100, $0.5 \%$ sodium deoxycholate, $1 \mathrm{mM}$ EDTA and $1 \mathrm{mM}$ PMSF) containing protease inhibitor cocktail (Roche, Basel, Switzerland) by vortex. $70 \mu \mathrm{g}$ of denatured protein was used in Western blotting and treated with the primary antibodies (HSD11B1 (Novusbio, Littleton, CO, USA; NBP1-69644, 1:1000), PCK1 (Cayman, Ann Arbor, MI, USA; 10004943, 1:1000), ALB (Dako, Glostrup, Denmark; A0001, 1:1000) and $\alpha$-tubulin (Cell signaling, Danvers, MA, USA; \#2144, 1:1000) and horseradish peroxidase (HRP)-conjugated rabbit secondary antibodies. After washing, the blots were exposed to enhanced chemiluminescence (ECL) reagent (Santa Cruz Biotech, Dallas, TX, USA) and then were developed.

\subsection{Statistical Analysis}

Data are presented as means \pm standard deviation (SD) and were analyzed by one-way or two-way ANOVA (GraphPad Prism Software, Inc., San Diego, CA, USA). $p<0.05$ was considered to indicate a statistically significant difference. 
Acknowledgments: This work was supported by the National Research Foundation of Korea (NRF) grant of Korean government (MEST) (No. 2017R1A2B2005031). Thank you for Han-Jin Park and Hyemin Kim working in the Korea Institute of Toxicology for providing the albumin antibody.

Author Contributions: Hee Young Kang and Eui-Bae Jeung conceived and designed the experiments; Hee Young Kang, Young-Kwon Choi and Yeon Ik Jeong performed the experiments; Hee Young Kang analyzed the data; Kyung-Chul Choi, Sang-Hwan Hyun, Woo-Suk Hwang and Eui-Bae Jeung contributed reagents/materials/analysis tools; Hee Young Kang wrote the paper.

Conflicts of Interest: The authors declare no conflict of interest.

\section{Abbreviations}

$\begin{array}{ll}\text { HSD11B1 } & \text { 11ß-hydroxysteroid dehydrogenase type 1 } \\ \text { TG } & \text { Transgenic } \\ \text { ER } & \text { Endoplasmic reticulum } \\ \text { SCNT } & \text { Somatic cell nuclear transfer } \\ \text { SV40LT } & \text { SV40 oncogene large T antigen } \\ \text { Pri11ßTG } & \text { primary HSD11B1-TG } \\ \text { SV11ßTG } & \text { SV40LT HSD11B1-TG } \\ \text { G6PT } & \text { Glucose-6-phosphate transporter } \\ \text { H6PD } & \text { Hexose-6-phosphate dehydrogenase } \\ \text { G6PC } & \text { Glucose-6 phosphatase catalytic subunit } \\ \text { PCK1 } & \text { Phosphoenolpyruvate carboxykinase 1 } \\ \text { GR } & \text { Glucocorticoid receptor } \\ \text { ALB } & \text { Albumin } \\ \text { AFP } & \alpha \text {-fetoprotein } \\ \text { SERPINA1 } & \text { Serpin peptidase inhibitor } \alpha 1 \text {-antitrypsin member } 1\end{array}$

\section{References}

1. Targher, G.; Bertolini, L.; Padovani, R.; Poli, F.; Scala, L.; Zenari, L.; Zoppini, G.; Falezza, G. Non-alcoholic fatty liver disease is associated with carotid artery wall thickness in diet-controlled type 2 diabetic patients. J. Endocrinol. Investig. 2006, 29, 55-60. [CrossRef] [PubMed]

2. Hewitt, K.N.; Walker, E.A.; Stewart, P.M. Minireview: Hexose-6-phosphate dehydrogenase and redox control of 11 $\beta$-hydroxysteroid dehydrogenase type 1 activity. Endocrinology 2005, 146, 2539-2543. [CrossRef] [PubMed]

3. Agarwal, A.K. Cortisol metabolism and visceral obesity: Role of $11 \beta$-hydroxysteroid dehydrogenase type I enzyme and reduced co-factor NADPH. Endocr. Res. 2003, 29, 411-418. [CrossRef] [PubMed]

4. White, P.C.; Mune, T.; Agarwal, A.K. 11ß-Hydroxysteroid dehydrogenase and the syndrome of apparent mineralocorticoid excess. Endocr. Rev. 1997, 18, 135-156. [CrossRef] [PubMed]

5. White, P.C.; Mune, T.; Rogerson, F.M.; Kayes, K.M.; Agarwal, A.K. Molecular analysis of 11 $\beta$-hydroxysteroid dehydrogenase and its role in the syndrome of apparent mineralocorticoid excess. Steroids 1997, 62, 83-88. [CrossRef]

6. Coderre, L.; Srivastava, A.K.; Chiasson, J.L. Role of glucocorticoid in the regulation of glycogen metabolism in skeletal muscle. Am. J. Physiol. 1991, 260, E927-E932. [PubMed]

7. Jung, E.M.; An, B.S.; Kim, Y.K.; Jeong, Y.H.; Hwang, W.S.; Jeung, E.B. Generation of porcine fibroblasts overexpressing 11 $\beta$-HSD1 with adipose tissue-specific aP2 promoter as a porcine model of metabolic syndrome. Mol. Med. Rep. 2013, 8, 751-756. [CrossRef] [PubMed]

8. Ge, R.; Huang, Y.; Liang, G.; Li, X. 11ß-hydroxysteroid dehydrogenase type 1 inhibitors as promising therapeutic drugs for diabetes: Status and development. Curr. Med. Chem. 2010, 17, 412-422. [CrossRef] [PubMed]

9. Kokubu, Y.; Asashima, M.; Kurisaki, A. Establishment and culture optimization of a new type of pituitary immortalized cell line. Biochem. Biophys. Res. Commun. 2015, 463, 1218-1224. [CrossRef] [PubMed]

10. Counter, C.M.; Avilion, A.A.; LeFeuvre, C.E.; Stewart, N.G.; Greider, C.W.; Harley, C.B.; Bacchetti, S. Telomere shortening associated with chromosome instability is arrested in immortal cells which express telomerase activity. EMBO J. 1992, 11, 1921-1929. [PubMed] 
11. Wright, W.E.; Shay, J.W. The two-stage mechanism controlling cellular senescence and immortalization. Exp. Gerontol. 1992, 27, 383-389. [CrossRef]

12. Ouellette, M.M.; McDaniel, L.D.; Wright, W.E.; Shay, J.W.; Schultz, R.A. The establishment of telomerase-immortalized cell lines representing human chromosome instability syndromes. Hum. Mol. Genet. 2000, 9, 403-411. [CrossRef] [PubMed]

13. Czimmerer, Z.; Hulvely, J.; Simandi, Z.; Varallyay, E.; Havelda, Z.; Szabo, E.; Varga, A.; Dezso, B.; Balogh, M.; Horvath, A.; et al. A versatile method to design stem-loop primer-based quantitative PCR assays for detecting small regulatory RNA molecules. PLoS ONE 2013, 8, e55168. [CrossRef] [PubMed]

14. Czegle, I.; Csala, M.; Mandl, J.; Benedetti, A.; Karadi, I.; Banhegyi, G. G6PT-H6PDH-11ßHSD1 triad in the liver and its implication in the pathomechanism of the metabolic syndrome. World J. Hepatol. 2012, 4, 129-138. [CrossRef] [PubMed]

15. Lu, N.Z.; Wardell, S.E.; Burnstein, K.L.; Defranco, D.; Fuller, P.J.; Giguere, V.; Hochberg, R.B.; McKay, L.; Renoir, J.M.; Weigel, N.L.; et al. International Union of Pharmacology. LXV. The pharmacology and classification of the nuclear receptor superfamily: Glucocorticoid, mineralocorticoid, progesterone, and androgen receptors. Pharmacol. Rev. 2006, 58, 782-797. [CrossRef] [PubMed]

16. Rhen, T.; Cidlowski, J.A. Antiinflammatory action of glucocorticoids-New mechanisms for old drugs. N. Engl. J. Med. 2005, 353, 1711-1723. [CrossRef] [PubMed]

17. Petersen, B.; Carnwath, J.W.; Niemann, H. The perspectives for porcine-to-human xenografts. Comp. Immunol. Microbiol. Infect. Dis. 2009, 32, 91-105. [CrossRef] [PubMed]

18. Liu, Y.; Nakagawa, Y.; Wang, Y.; Sakurai, R.; Tripathi, P.V.; Lutfy, K.; Friedman, T.C. Increased glucocorticoid receptor and $11 \beta$-hydroxysteroid dehydrogenase type 1 expression in hepatocytes may contribute to the phenotype of type 2 diabetes in $d b / d b$ mice. Diabetes 2005, 54, 32-40. [CrossRef] [PubMed]

19. Lemke, U.; Krones-Herzig, A.; Berriel Diaz, M.; Narvekar, P.; Ziegler, A.; Vegiopoulos, A.; Cato, A.C.; Bohl, S.; Klingmuller, U.; Screaton, R.A.; et al. The glucocorticoid receptor controls hepatic dyslipidemia through Hes1. Cell Metab. 2008, 8, 212-223. [CrossRef] [PubMed]

20. Garcovich, M.; Zocco, M.A.; Gasbarrini, A. Clinical use of albumin in hepatology. Blood Transfus. 2009, 7, 268-277. [PubMed]

21. Bandiera, S.; Pfeffer, S.; Baumert, T.F.; Zeisel, M.B. miR-122-A key factor and therapeutic target in liver disease. J. Hepatol. 2015, 62, 448-457. [CrossRef] [PubMed]

22. Hsu, S.H.; Wang, B.; Kota, J.; Yu, J.; Costinean, S.; Kutay, H.; Yu, L.; Bai, S.; La Perle, K.; Chivukula, R.R.; et al. Essential metabolic, anti-inflammatory, and anti-tumorigenic functions of miR-122 in liver. J. Clin. Investig. 2012, 122, 2871-2883. [CrossRef] [PubMed]

23. Tsai, W.C.; Hsu, S.D.; Hsu, C.S.; Lai, T.C.; Chen, S.J.; Shen, R.; Huang, Y.; Chen, H.C.; Lee, C.H.; Tsai, T.F.; et al. MicroRNA-122 plays a critical role in liver homeostasis and hepatocarcinogenesis. J. Clin. Investig. 2012, 122, 2884-2897. [CrossRef] [PubMed]

24. Mizejewski, G.J. Levels of $\alpha$-fetoprotein during pregnancy and early infancy in normal and disease states. Obstet. Gynecol. Surv. 2003, 58, 804-826. [CrossRef] [PubMed]

25. Yuen, M.F.; Lai, C.L. Screening for hepatocellular carcinoma: Survival benefit and cost-effectiveness. Ann. Oncol. 2003, 14, 1463-1467. [CrossRef] [PubMed]

26. Chou, J.Y.; Mano, T.; Feldman, M. Inhibition of synthesis of $\alpha$-fetoprotein by glucocorticoids in cultured hepatoma cells. J. Cell Biol. 1982, 93, 314-317. [CrossRef] [PubMed]

27. Drugan, A.; Subramanian, M.G.; Johnson, M.P.; Evans, M.I. Amniotic fluid cortisol and $\alpha$-fetoprotein in normal and aneuploid pregnancies. Fetal Ther. 1988, 3, 192-197. [CrossRef] [PubMed]

28. Illyes, G.; Luczay, A.; Benyo, G.; Kalman, A.; Borka, K.; Koves, K.; Racz, K.; Tulassay, T.; Schaff, Z. Cushing's syndrome in a child with pancreatic acinar cell carcinoma. Endocr. Pathol. 2007, 18, 95-102. [CrossRef] [PubMed]

29. Schiodt, F.V.; Ostapowicz, G.; Murray, N.; Satyanarana, R.; Zaman, A.; Munoz, S.; Lee, W.M. Alpha-fetoprotein and prognosis in acute liver failure. Liver Transpl. 2006, 12, 1776-1781. [CrossRef] [PubMed]

30. Saunders, D.N.; Tindall, E.A.; Shearer, R.F.; Roberson, J.; Decker, A.; Wilson, J.A.; Hayes, V.M. A novel SERPINA1 mutation causing serum $\alpha 1$-antitrypsin deficiency. PLoS ONE 2012, 7, e51762. [CrossRef] [PubMed] 
31. Gettins, P.G. Serpin structure, mechanism, and function. Chem. Rev. 2002, 102, 4751-4804. [CrossRef] [PubMed]

32. Kushner, I. Regulation of the acute phase response by cytokines. Perspect. Biol. Med. 1993, 36, 611-622. [CrossRef] [PubMed]

33. DeMeo, D.L.; Silverman, E.K. $\alpha 1$-antitrypsin deficiency. 2: Genetic aspects of $\alpha 1$-antitrypsin deficiency: Phenotypes and genetic modifiers of emphysema risk. Thorax 2004, 59, 259-264. [CrossRef] [PubMed]

34. Cowden, D.I.; Fisher, G.E.; Weeks, R.L. A pilot study comparing the purity, functionality and isoform composition of $\alpha 1$-proteinase inhibitor (human) products. Curr. Med. Res. Opin. 2005, 21, 877-883. [CrossRef] [PubMed]

35. Jonigk, D.; Al-Omari, M.; Maegel, L.; Muller, M.; Izykowski, N.; Hong, J.; Hong, K.; Kim, S.H.; Dorsch, M.; Mahadeva, R.; et al. Anti-inflammatory and immunomodulatory properties of $\alpha 1$-antitrypsin without inhibition of elastase. Proc. Natl. Acad. Sci. USA 2013, 110, 15007-15012. [CrossRef] [PubMed]

36. Elenkov, I.J. Glucocorticoids and the Th1/Th2 balance. Ann. N. Y. Acad. Sci. 2004, 1024, 138-146. [CrossRef] [PubMed]

37. Parent, R.; Durantel, D.; Lahlali, T.; Salle, A.; Plissonnier, M.L.; DaCosta, D.; Lesca, G.; Zoulim, F.; Marion, M.J.; Bartosch, B. An immortalized human liver endothelial sinusoidal cell line for the study of the pathobiology of the liver endothelium. Biochem. Biophys. Res. Commun. 2014, 450, 7-12. [CrossRef] [PubMed]

38. Kim, C.D.; Lee, M.H.; Roh, S.S. Identification of androgen-regulated genes in SV40-transformed human hair dermal papilla cells. J. Dermatol. Sci. 2003, 32, 143-149. [CrossRef]

39. Varkonyi-Gasic, E.; Hellens, R.P. Quantitative stem-loop RT-PCR for detection of microRNAs. Methods Mol. Biol. 2011, 744, 145-157. [PubMed]

(C) 2017 by the authors. Licensee MDPI, Basel, Switzerland. This article is an open access article distributed under the terms and conditions of the Creative Commons Attribution (CC BY) license (http:/ / creativecommons.org/licenses/by/4.0/). 